\title{
Glucocorticoids induce long-lasting effects in neural stem cells resulting in senescence-related alterations
}

\author{
R Bose ${ }^{1}$, M Moors ${ }^{1}$, R Tofighi ${ }^{1}$, A Cascante ${ }^{1}, 0$ Hermanson ${ }^{1}$ and S Ceccatelli,
}

Alterations in intrauterine programming occurring during critical periods of development have adverse consequences for wholeorgan systems or individual tissue functions in later life. In this paper, we show that rat embryonic neural stem cells (NSCs) exposed to the synthetic glucocorticoid dexamethasone (Dex) undergo heritable alterations, possibly through epigenetic mechanisms. Exposure to Dex results in decreased NSC proliferation, with no effects on survival or differentiation, and changes in the expression of genes associated with cellular senescence and mitochondrial functions. Dex upregulates cell cycle-related genes p16 and p21 in a glucocorticoid receptor(GR)-dependent manner. The senescence-associated markers high mobility group (Hmg) A1 and heterochromatin protein 1 (HP1) are also upregulated in Dex-exposed NSCs, whereas Bmi1 (polycomb ring finger oncogene) and mitochondrial genes Nd3 (NADH dehydrogenase 3) and Cytb (cytochrome b) are downregulated. The concomitant decrease in global DNA methylation and DNA methyltransferases (Dnmts) suggests the occurrence of epigenetic changes. All these features are retained in daughter NSCs (never directly exposed to Dex) and are associated with a higher susceptibility to oxidative stress, as shown by the increased occurrence of apoptotic cell death on exposure to the redox-cycling reactive oxygen species (ROS) generator 2,3-dimethoxy-1-naphthoquinone (DMNQ). Our study provides novel evidence for programming effects induced by glucocorticoids (GCs) on NSCs and supports the idea that fetal exposure to endogenous or exogenous GCs is likely to result in long-term consequences that may predispose to neurodevelopmental and/or neurodegenerative disorders.

Cell Death and Disease (2010) 1, e92; doi:10.1038/cddis.2010.60; published online 4 November 2010

Subject Category: Neuroscience

Epidemiological and experimental studies have shown that alterations in the intrauterine programming occurring during critical periods of development may have adverse consequences in later life. ${ }^{1}$ Morphometrical analyses have correlated certain patterns of intrauterine growth, particularly growth retardation, to specific postnatal outcomes with increased incidence of cardiovascular, metabolic, renal and endocrine disorders. ${ }^{2}$ Interestingly, recent clinical studies have shown a correlation between intrauterine growth retardation and the development of attention-deficit hyperactive disorder (ADHD) ${ }^{3}$ and depression. ${ }^{4}$ Hormones such as glucocorticoids (GCs) have a critical role in intrauterine programming. During development, GCs control fetal growth of all tissues and organs, and have a critical role in intrauterine programming, ${ }^{5}$ exerting their action by changing the expression of receptors, enzymes, ion channels, transporters, various growth factors, cytoarchitectural proteins, binding proteins and components of intracellular signalling pathways. They can function directly on genes and/or indirectly, affecting, for example, the bioavailability of other hormones. ${ }^{6}$
Most of the prenatal challenges known to have programming effects have been associated with high levels of GCs in utero. ${ }^{1}$ Fetal exposure to elevated GC levels can occur when exogenous GCs are administered for therapeutic purposes or in response to severe maternal stress. Normally, the fetus is protected from maternal GCs by placental 11 beta hydroxysteroid dehydrogenase type 2 (11 $\beta$-HSD2). However, high maternal levels exceeding the limit of placental $11 \beta$-HSD2 or pathological conditions impairing placental functions might lead to fetal exposure to excess GCs. ${ }^{5}$

It is well established that GCs, although critical for adaptation to stress, can have adverse effects on the nervous system when secreted in excess. ${ }^{7,8}$ Animal models have provided compelling evidence that fetal exposure to high levels of GCs alter brain development, causing persistent structural and functional changes and also affecting fetal neurogenesis. ${ }^{9-11}$ Certain regions of the adult brain retain neurogenic potential ${ }^{12}$ and several studies have shown that, in the adult hippocampus, GCs negatively affect neurogenesis. ${ }^{13,14}$ Relevantly, the effects of early exposure to an

\footnotetext{
${ }^{1}$ Department of Neuroscience, Karolinska Institutet, Retziusväg 8, Stockholm S-17177, Sweden

*Corresponding author: S Ceccatelli, Department of Neuroscience, Karolinska Institutet, Retziusväg 8, Stockholm S-17177, Sweden. Tel: + 46852487586 ; Fax: + 468333 864; E-mail: Sandra.Ceccatelli@ki.se

Keywords: neurodevelopment; epigenetic; fetal programming

Abbreviations: NSCs, neural stem cells; ADHD, attention-deficit hyperactive disorder; GCs, glucocorticoids; $11 \beta$-HSD2, 11 beta hydroxysteroid dehydrogenase type 2; Dex, dexamethasone; P1, parent NSCs; D, daughter cells; q-PCR, quantitative real-time PCR; GR, glucocorticoid receptor; Hmg, high mobility group; SAHF, senescence-associated heterochromatin foci; HP1 $\gamma$, heterochromatin protein 1 gamma; Nd3, NADH dehydrogenase 3; Cytb, cytochrome b; Dnmts, DNA methyltransferases; Bmi1, polycomb ring finger oncogene; Hprt, hypoxanthine phosphoribosyltransferase; DMNQ, 2,3-dimethoxy-1,4-naphthoquinone; ROS, reactive oxygen species; GREs, GC responsive elements; PF, paraformaldehyde; OD, optical density; carboxy- $\mathrm{H}_{2} \mathrm{DCFDA}, 5$-(and-6)-carboxy-2' $7^{\prime}$-dichlorodihydrofluorescein diacetate; PBS, phosphate-buffered saline; RT, room temperature; BSA, bovine serum albumin

Received 28.7.10; revised 12.8.10; accepted 17.8.10; Edited by A Verkhrasky
} 
increased level of GCs, as induced by prenatal stress, are long lasting, resulting in reduced neurogenesis in later life in rodents and non-human primates. ${ }^{9,15}$

In previous studies, we observed that prenatal exposure to high levels of synthetic GC dexamethasone (Dex) induces long-lasting alterations in rat neuronal cells characterized by an increased susceptibility to oxidative stress, ${ }^{16}$ which we also observed in adult NSCs. ${ }^{17}$ All together, the data pointed to a programming effect of Dex, which induced long-term changes in cells never directly exposed to it. In light of the central role that NSCs have in the developing and also adult nervous system, we designed the present study to investigate the molecular mechanisms responsible for the long-lasting effects induced by GCs in primary cultures of embryonic cortical NSCs.

\section{Results}

GC-induced effects were investigated in parent NSCs (P1) exposed to $1 \mu \mathrm{M}$ Dex for $48 \mathrm{~h}$, and in daughter cells (D) from passage 2 (D2) and 3 (D3), which were never directly exposed to Dex (see Figure 1 for details).

\section{Dex decreases proliferation of NSCs without interfering} with cell viability or differentiation. Live cell imaging revealed that Dex-exposed $\mathrm{P} 1$ had more processes and tended to grow disjointed, whereas control cells grew in clusters with tight cell-cell contacts (Figure $2 a$ and b). In addition, Dex exposure led to a significant reduction in total cell number, as observed in P1 cells, as well as in D2 and D3 cells (Figure 2c), without a concomitant increase in necrotic (data not shown) or apoptotic cells (Supplementary Figure S1). As shown by the immunocytochemical staining for the proliferation marker Ki67 (green colour), Dex exposure significantly decreased proliferation in $\mathrm{P} 1$ (Figure $3 a$ and $b$ ) and this effect persisted in $D$ (Figure $3 c$ ).

To clarify whether the reduction in cell proliferation was associated with an increase in differentiation, we performed immunocytochemical analyses in control and Dex P1, D2 and D3 cells using markers for NSCs (nestin, green colour), early neurons (TuJ1, green colour) and glia progenitor cells (GFAP, red colour). Nearly $100 \%$ of the control, as well as Dex P1 (Figure 3d and e), D2 and D3 cells, were nestin positive, whereas no TuJ1- or GFAP-positive cells were detected at any passage (Supplementary Figure S2).

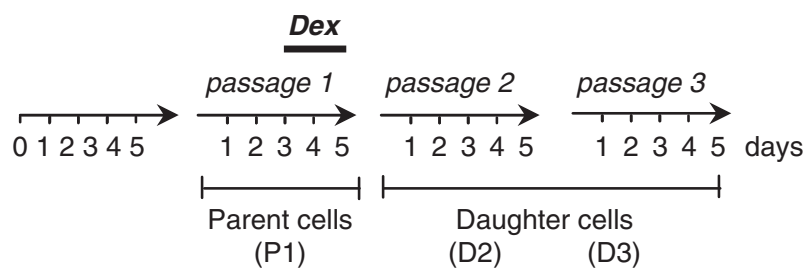

Figure 1 Experimental model. After cortice dissection and cell plating, NSCs were cultured for 5 days before passaging in order to obtain parent cells (P1). After 3 days in culture, P1 cells were exposed to Dex $(1 \mu \mathrm{M})$ for $48 \mathrm{~h}$. To investigate the long-lasting (heritable) effects of Dex, P1 NSCs were passaged to get daughter cells (D). D2 = daughter NSCs from passage 2; D3= daughter NSCs from passage 3
Dex increases gene expression of cell-cycle inhibitors and hallmarks of senescence. Gene expression analyses by quantitative real-time PCR (q-PCR) in P1 cells revealed a significant Dex-induced upregulation of the cell-cycle regulating genes $p 16$ (fold change $1.98 \pm 0.08$ ) (Figure $4 a$ ) and $p 21$ (fold change $2.14 \pm 0.15$ ) (Figure $4 \mathrm{~b}$ ) that persisted in D2 ( $p 16$ fold change $2.02 \pm 0.2, p 21$ fold change $1.75 \pm 0.06$ ) and D3 NSCs ( $p 16$ fold change $1.89 \pm 0.13$, p21 fold change $2.17 \pm 0.31$ ). Preexposure to the glucocorticoid receptor (GR) antagonist Mifepristone (RU486) significantly inhibited the $p 16$ (fold change $1.23 \pm 0.05$ ) (Supplementary Figure S3A) and p21 (fold change 1.42 \pm 0.07 ) (Supplementary Figure S3B) upregulation, without altering $G R$ expression (fold change $0.92 \pm 0.2$ ) (Supplementary Figure S3C), pointing to a GR-mediated mechanism. siRNA nucleofection to knock down GR (efficiency about $50-70 \%$, fold change $0.3 \pm 0.01$ ) (Supplementary Figure S3D) blocked Dex-induced $p 16$ (fold change 1.45 \pm 0.13 ) (Figure 4c) and p21 (fold change 1.32 \pm 0.09 ) (Figure 4d) upregulation significantly.

In light of the fact that p16 and p21 upregulation has been linked to cellular senescence, we analysed Dex-induced effects on the expression of senescence markers by q-PCR and immunocytochemistry. Interestingly, Dex repressed Bmi1 (polycomb ring finger oncogene) expression in P1 (fold change $0.33 \pm 0.03$ ), D2 (fold change $0.32 \pm 0.03$ ) and D3 NSCs (fold change $0.46 \pm 0.06$ ) (Figure 5a) and upregulated high mobility group A1 (Hmga1) in D2 (fold change $2.09 \pm 0.26$ ) and D3 cells (fold change 2.06 \pm 0.22 ) (Figure 5b). In contrast, we did not detect significant changes in Hmga2 expression (P1 fold change $1.36 \pm 0.1$, D2 fold change $0.89 \pm 0.03$, D3 fold change 1.12 \pm 0.15 ) (Supplementary Figure S4). In addition, Dex led to an enrichment of heterochromatin protein 1 gamma (HP1 $\gamma$ ) (green colour) in socalled senescence-associated heterochromatin foci (SAHF) (Figures $5 c$ and $d$ ).

Dex alters the expression of mitochondrial respiratory chain genes. Cellular senescence has been associated with impaired mitochondrial functions. Interestingly, gene expression analyses by q-PCR showed a long-lasting downregulation of mitochondrial genes $\mathrm{NADH}$ dehydrogenase 3 (Nd3) (P1 fold change $0.41 \pm 0.06$, D2 fold change $0.41 \pm 0.03$, D3 fold change $0.43 \pm 0.05$ ) (Figure 6a) and cytochrome b (Cytb) (P1 fold change $0.47 \pm 0.05$, D2 fold change $0.48 \pm 0.04$, D3 fold change $0.45 \pm 0.03$ ) (Figure 6B).

Dex modifies global DNA methylation. Methylation measurements revealed a significant decrease in global DNA methylation in P1 $(\sim 33 \%)$ and D2 ( 40\%) NSCs (Figure 7a) associated with a decreased expression in DNA methyltransferases (Dnmts). In parent cells, a significant downregulation in Dnmt1 (fold change 0.53 \pm 0.02 ), Dnmt3a (fold change $0.62 \pm 0.01$ ) and Dnmt3b mRNA levels (fold change $0.5 \pm 0.01$ ) was detected (Figure $7 \mathrm{~b}$ ), whereas $D$ showed a decrease in Dnmt1 (fold change $0.69 \pm 0.01$ ) and Dnmt3a expression (fold change $0.68 \pm 0.01$ ) (Figure $7 \mathrm{c}$ ).

Dex induces a long-lasting susceptibility to oxidative stress. We then wanted to test the hypothesis that the longlasting senescence and mitochondrial alterations would be 


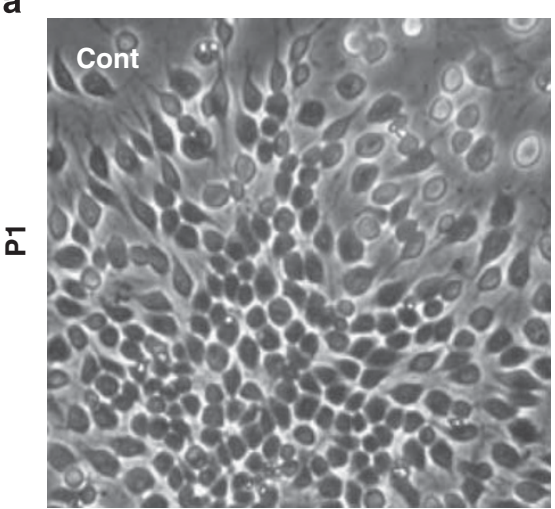

b

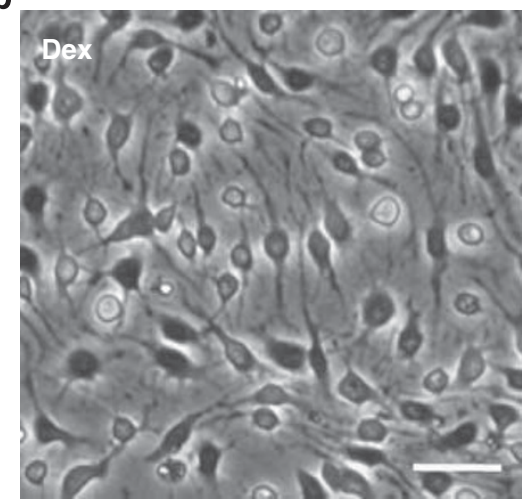

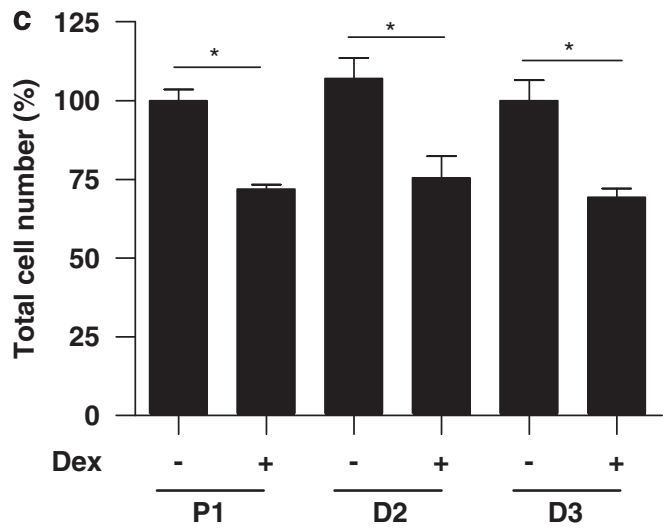

Figure 2 Dex exposure decreases NSC proliferation. Phase contrast images of control (a) and Dex-exposed ( $1 \mu \mathrm{M}$ Dex for $48 \mathrm{~h})(\mathbf{b}) \mathrm{P} 1 \mathrm{NSCs}$. Scale bar $=50 \mu \mathrm{m}$. (c) Total cell number (expressed as percentage from cell counts of control cells) of parent (P1) and D2 and D3 cells. Values are shown as mean \pm S.E.M. ${ }^{*} P<0.05$

associated with a higher vulnerability to oxidative stress. We challenged daughter NSCs with the oxidative stress inducer 2,3-dimethoxy-1,4-naphthoquinone (DMNQ, $3 \mu \mathrm{M}$ ) and observed an increased reactive oxygen species (ROS) production in Dex-NSCs, compared with control cells (Figure 8a), which were associated with a higher incidence of cell death, as shown by the twofold increase in the number of apoptotic NSCs (Figure 8b).

\section{Discussion}

The novelty of our findings is the demonstration that, in NSCs, GCs induce heritable alterations, including a decrease in proliferation and changes in the expression of genes related to senescence and mitochondrial functions. The decrease in global DNA methylation suggests that epigenetic mechanisms may have a critical role in the onset of Dex-induced effects. In addition, both parent cells and D exhibited an increased susceptibility to oxidative stress. All together, the data open new translational perspectives. Considering the central role that NSCs have in the developing nervous system, it is conceivable that, in vivo, the alterations induced by GCs on cortical NSCs can negatively affect neurogenesis with a subsequent unfavourable functional outcome in terms of cognitive abilities. ${ }^{18,19}$ Both human and animal studies have demonstrated that disruptions in prenatal and early postnatal brain development can cause mental retardation and cognitive dysfunctions, ${ }^{20}$ and disruption of developmental neurogenesis has been linked to schizophrenia. ${ }^{21}$ It is well established that NSCs are also present in the adult nervous system, where they may have a role in learning, memory and in the response to injuries. ${ }^{12}$ Thus, the longlasting impairment induced by GCs in NSCs can have negative consequences in adulthood as well.

In terms of cellular mechanisms, the decrease in NSC number induced by Dex that we observed was not associated with an increase in cell death or differentiation, clearly pointing to an alteration in the proliferation rate, in agreement with Sundberg et al. ${ }^{22}$ Gene expression analyses revealed an upregulation of cell-cycle regulating genes $p 16$ and $p 21$, which represent potent inhibitors of cyclin-dependent kinases and D-type cyclins in various cell types, thereby leading to cell cycle arrest. $^{23,24}$ By blocking GR with the non-selective antagonist Mifepristone or through knockdown experiments targeting GR expression, we could prevent the upregulation of both $p 16$ and $p 21$. Gene expression regulation by GR can mechanistically occur in two distinct ways: (1) the ligandactivated GR interacts directly with GC-responsive elements (GREs) that are present in promoter regions of target genes; (2) GR-mediated stimulation of gene expression takes place by GR interaction with other DNA-bound proteins, without direct binding to the DNA itself or by interaction with other molecules involved in intracellular signalling. ${ }^{25}$ For $p 21$, GC-responsive regions have been identified in the rat 
a

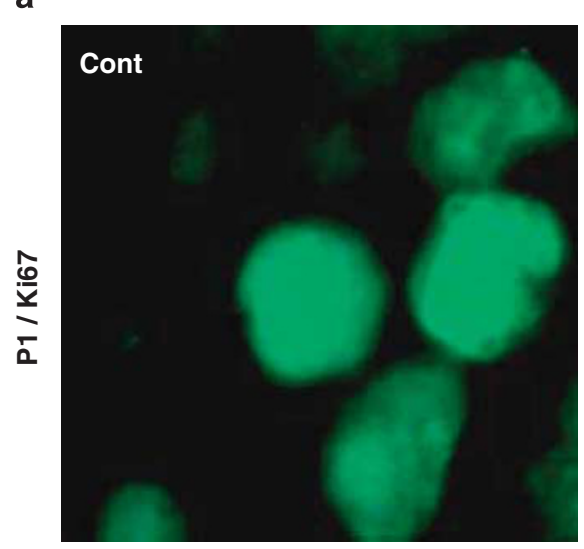

b
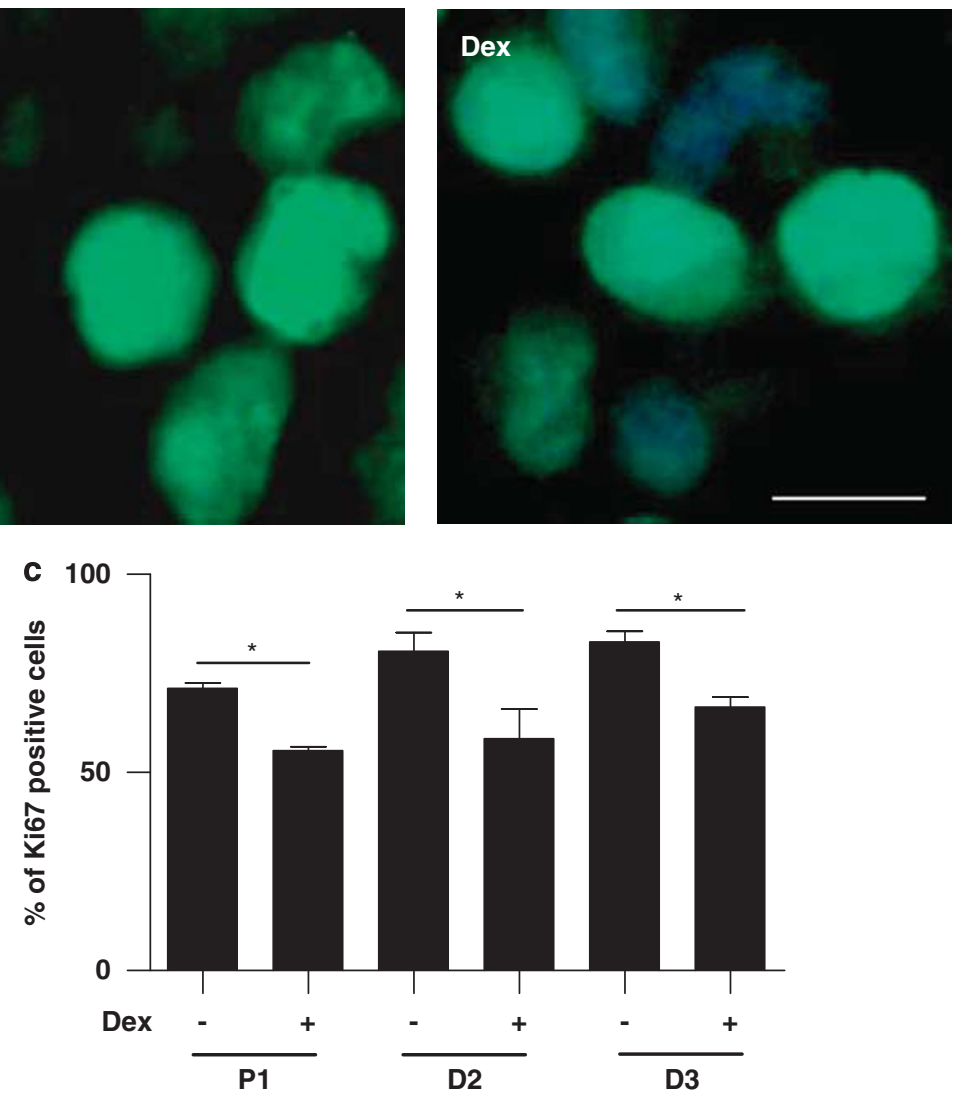

e
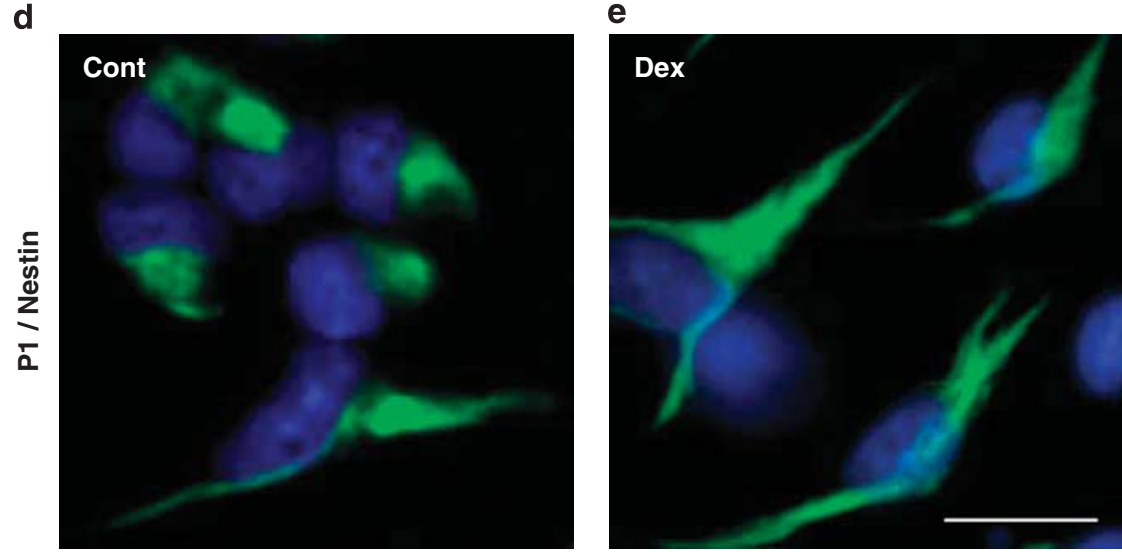

Figure 3 Dex decreases the number of Ki67-positive NSCs without changing the percentage of nestin-positive cells. Micrographs show Ki67-positive cells (green colour) $(\mathbf{a}, \mathbf{b})$ and the percentage of positive cells is given in (c). Cytoplasmatic staining for nestin (green colour) in control (d) and Dex (e) NSCs. Nuclei are stained with Hoechst 33342 (blue colour). Values are shown as mean \pm S.E.M. ${ }^{\star} P<0.05$. Scale bar $=50 \mu \mathrm{m}$

promoter sequence that requires the presence of a $C / E B P$ DNA binding site and the expression of a functional $\mathrm{C} / \mathrm{EBP} \alpha$ transcription factor. ${ }^{26,27}$ Thus, Dex-induced $p 21$ upregulation might be occurring via functional protein interactions between $\mathrm{GR}$ and $\mathrm{C} / \mathrm{EBP} \alpha$ in a tethering mechanism.

The upregulation of p16 and p21 has been linked to cellular and in vivo senescence, ${ }^{28,29}$ and our data point to Dex promoting senescence in NSCs, an effect that is passed from parent cells to $D$. Cellular senescence is accompanied by SAHFs containing a variety of chromatin proteins, such as K9-methylated histone H3, macro H2A, HP1 and HMGA proteins. ${ }^{30}$ Gene expression analyses of $\mathrm{Hmga} 1$ and protein analyses via immunocytochemistry against HP1 $\gamma$ showed that both senescence markers are increased in daughter Dex-NSCs. Further, we also detected a downregulation of $B m i 1$ that is also assumed to be an indicator of senescence based on the fact that normal embryonic fibroblasts from Bmi1-/- mice show a premature senescence phenotype that correlates with an increased expression of $p 16 .^{31}$

Cellular senescence is associated with mitochondrial dysfunctions as shown in several different cell types, including fibroblasts, epithelial, mesothelial and hippocampal cells. ${ }^{32-35}$ 

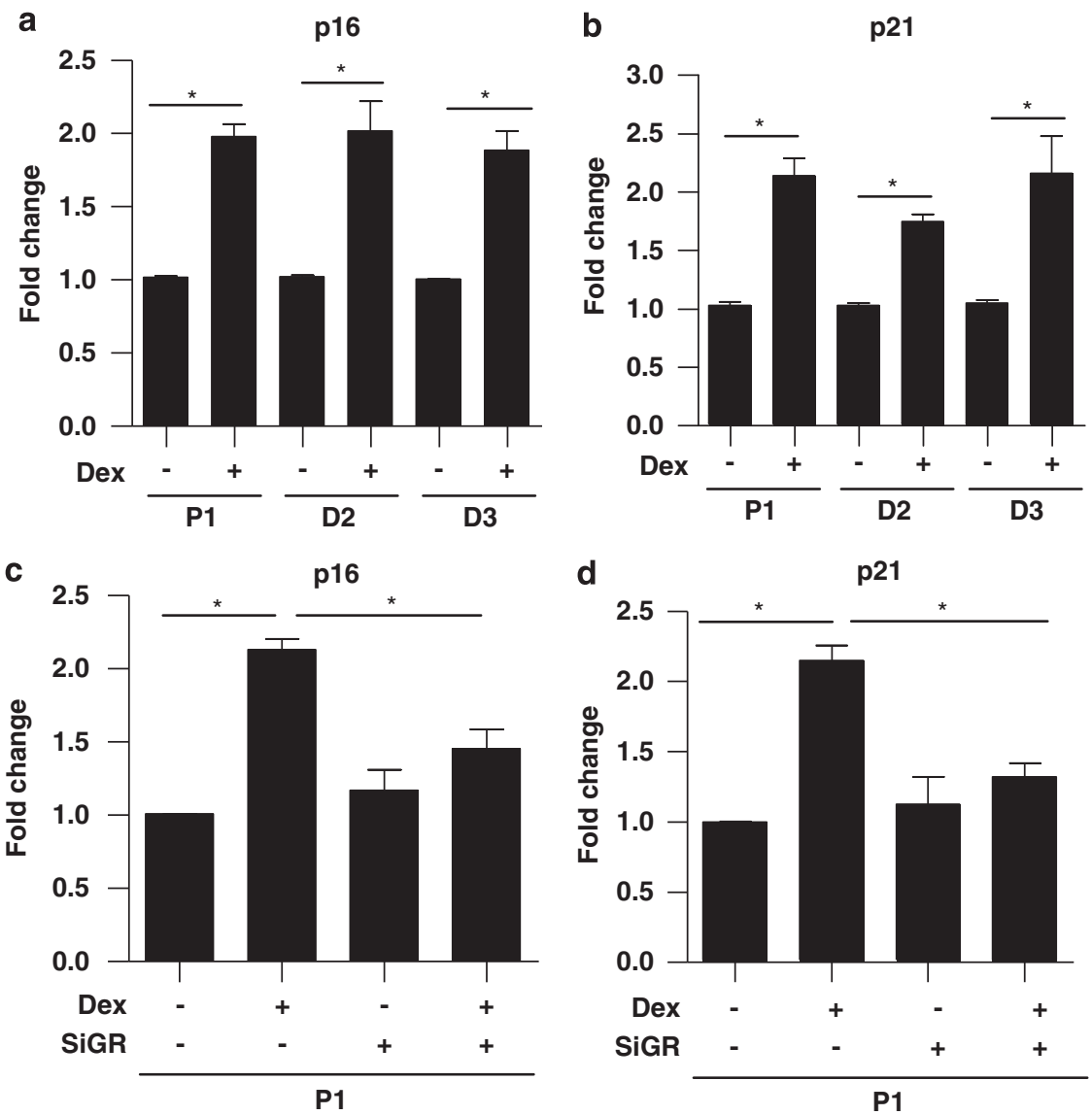

Figure 4 The long-lasting upregulation of $p 16$ and $p 21$ expression is GR dependent. Gene expression analyses by q-PCR showed that $p 16$ (a) and $p 21$ (b) genes were upregulated in P1 NSCs (immediately after $48 \mathrm{~h}$ Dex exposure), as well as in D2 and D3 NSCs (24 h after the respective passaging). siRNA nucleofection to knock down GR blocked the Dex-induced $p 16$ (c) and $p 21$ (d) upregulation. Relative quantification expression values were calculated by the arithmetic equation $2^{-\Delta \Delta C T}$. The amount of target genes was normalized to Hprt and the relative increase was equal to $2^{-\Delta(\Delta C T D e x-\Delta C T c o n t r o l)}$. ${ }^{*} P<0.05$

Particularly, increased ROS content and decreased mitochondrial energetic competences to produce ATP are two major events underlined in the mitochondrial hypothesis of ageing. ${ }^{36}$ Mitochondria are known to be a primary target of GCs, as the mitochondrial genome contains six putative GREs. Gel shift analysis proved binding of the GR to the mitochondrial genome after GC challenge, ${ }^{37}$ which results in transcriptional regulation of several mitochondria-encoded genes. ${ }^{38}$ In our cell model, we observed a transcriptional repression of the mitochondrial-respiratory chain enzymes of complex I (Nd3) and complex III (Cytb) induced by Dex in parent cells and D. Notably, a study on adrenalectomized rats has shown that several mitochondrial genes, including Cytb, are $\mathrm{GC}$ responsive and are downregulated in response to low doses of corticosterone. ${ }^{38}$

In our studies, all the effects induced by Dex persisted from parent cells to $D$. The changes in global DNA methylation accompanied by a decreased expression of Dnmt1, Dnmt3a and Dnmt3b suggest that the heritable effects induced by Dex might be due to epigenetic reprogramming of NSCs. The identification of gene-specific alterations in DNA methylation is the aim of ongoing studies.

In agreement with our previous data, ${ }^{16,17}$ Dex induces a long-lasting increased susceptibility to oxidative stress, as shown by the higher number of cells undergoing apoptosis, associated with higher levels of intracellular ROS. However, the long-lasting alterations induced by GCs do not interfere with the viability of NSCs, unless they are challenged by an adverse stimulus, such as oxidative stress.

In conclusion, our data provide novel evidence for programming effects induced by Dex in NSCs and support the idea that conditions associated with fetal exposure to high levels of GCs are likely to have long-term consequences that may predispose to neurodevelopmental disorders and/or to neurodegenerative processes.

\section{Materials and Methods}

Embryonic cortical NSCs culture and exposure procedures. Primary cultures of NSCs were prepared as previously described. ${ }^{39,40}$ Cells were obtained from embryonic cortices $(n=6-8 /$ cell prepartion) dissected in HBSS (Life technologies, Carlsbad, CA, USA) from timed pregnant Sprague Dawley rats $(n=20)$ (Harlan Laboratories, Harlan, The Netherlands) at E15 (the day of copulatory plug defined as E0). The tissue was mechanically dispersed, and meninges and larger cell clumps were allowed to sediment for $10 \mathrm{~min}$. The cells were plated at a density of $40.000 / \mathrm{cm}^{2}$ on dishes precoated with poly-L-ornithine and fibronectin (both from Sigma-Aldrich, St. Louis, $\mathrm{MO}$, USA; Stockholm, Sweden). Cells were maintained in enriched $\mathrm{N}_{2}$ medium with $10 \mathrm{ng} / \mathrm{ml}$ of basic fibroblast growth factor (R\&D systems, Minneapolis, MN, USA) added every $24 \mathrm{~h}$ and medium changed every alternate day to keep the cells in an 

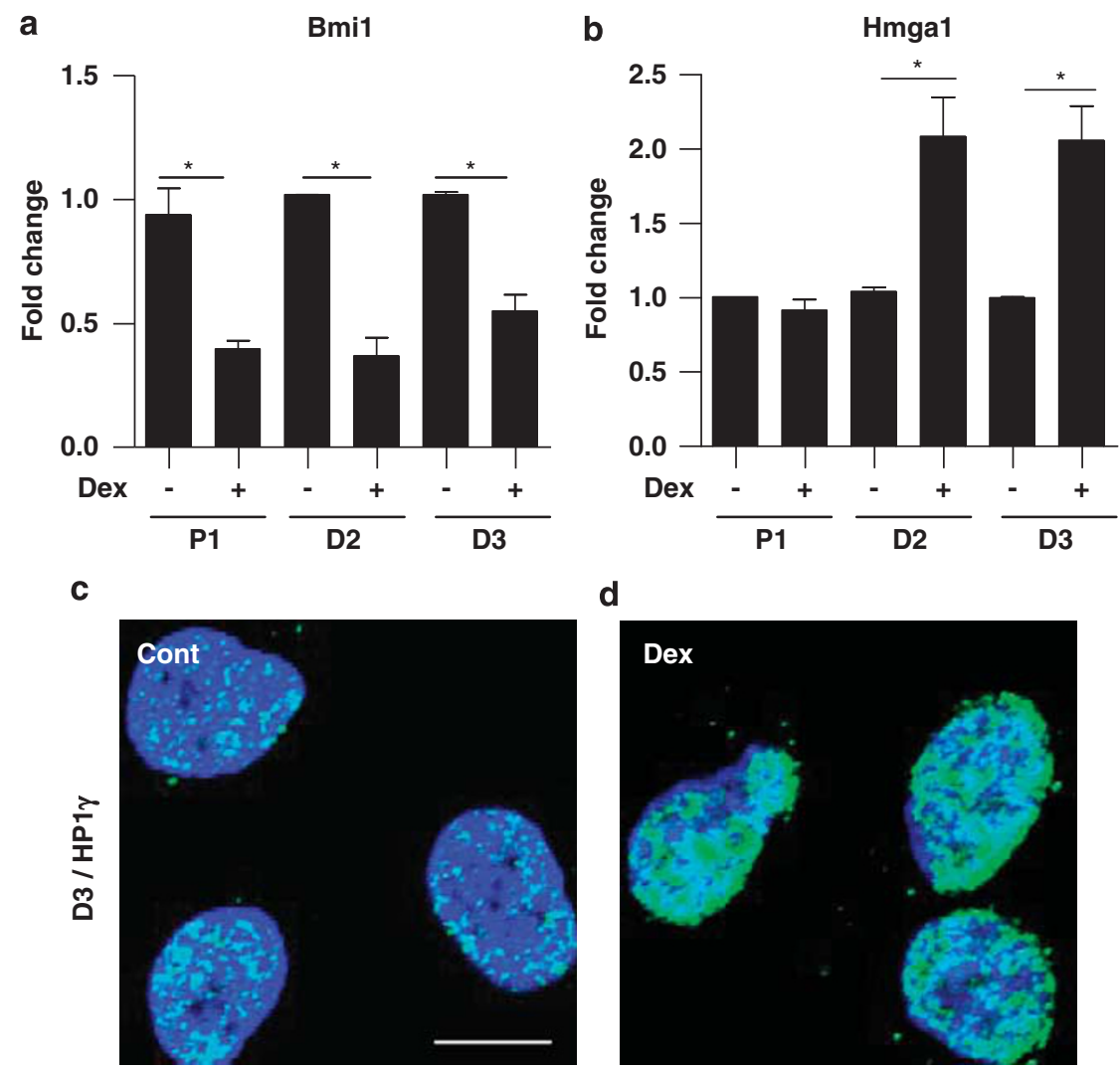

Figure 5 Dex induces the expression of senescence markers in NSCs. q-PCR analyses showed that the expression level of Bmi1 (a) was significantly decreased in P1 NSCs $48 \mathrm{~h}$ after Dex exposure, as well as in D2 and D3 NSCs $24 \mathrm{~h}$ after the respective passaging. The increased expression of $H m g a 1 \mathrm{mRNA}$ (b) was observed only in D2 and D3 cells. Confocal images of control (c) and Dex (d) NSCs showing an enrichment of heterochromatin protein 1 gamma (HP1 $\gamma$ ) (green colour) induced by Dex. Nuclei were counterstained with Hoechst 33342 (blue colour). ${ }^{*} P<0.05$, Scale bar $=50 \mu \mathrm{m}$
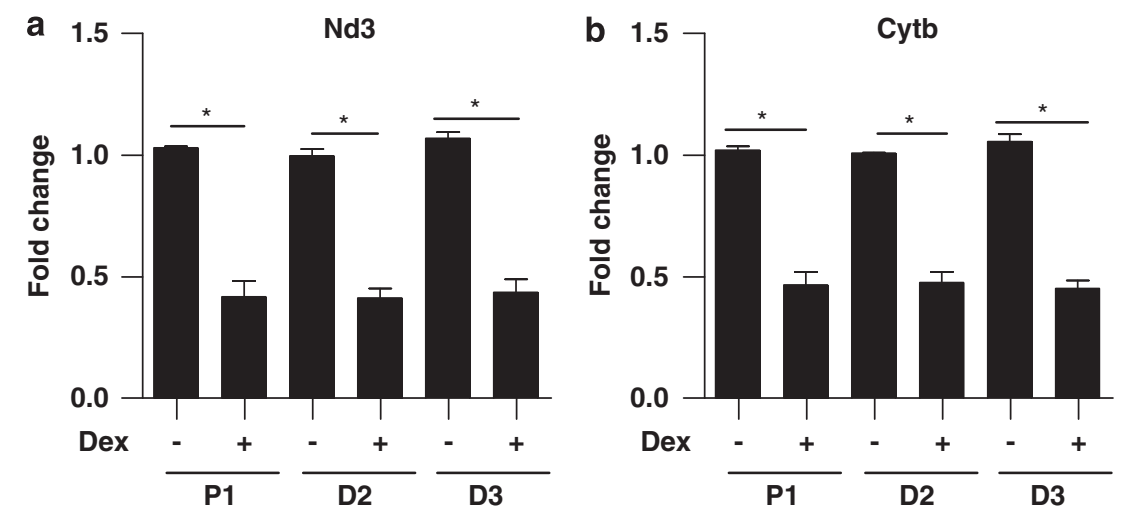

Figure 6 Dex alters the expression of mitochondrial genes. A long-lasting significant decrease of Nd3 (a) and Cytb (b) mRNA expression was induced by Dex in NSCs. Values are shown as mean \pm S.E.M. ${ }^{*} P<0.05$

undifferentiated and proliferative state. Cells were passaged by detaching through scraping in HBSS. Thereafter, the cells were gently mixed in $\mathrm{N}_{2}$ medium, counted, and plated at a desired density. With these culture conditions, doubling time of NSCs was $\sim 20 \mathrm{~h}$. For counting and evaluation of cell viability, cells were harvested in HBSS, stained with $0.4 \%$ Trypan blue solution (Sigma-Aldrich) and analysed under a phase-contrast microscope using a Neubauer improved counting chamber. Cells with a damaged cell membrane (necrotic cells) stained blue, whereas cells with intact plasma membrane (healthy or apoptotic cells) remained unstained. To investigate Dex long-lasting effects, we exposed P1 NSCs (see Figure 1) to Dex $(1 \mu \mathrm{M})$ for $48 \mathrm{~h}$. P1 cells were harvested at the end of the exposure to Dex. Heritable effects were investigated in D2 and D3 NSCs (see Figure 1) at different time points, depending on the type of analysis.

For the investigation of GR-dependent mechanisms, P1 NSCs were incubated with $200 \mathrm{nM}$ Mifepristone for $30 \mathrm{~min}$ before exposure to Dex. To induce oxidative stress, we used DMNQ (Calbiochem, Darmstadt, Germany). D2 NSCs (72 h after passaging) were exposed to $3 \mu \mathrm{M}$ DMNQ for up to $24 \mathrm{~h}$. All experiments were performed in triplicate and repeated at least five times.

Immunocytochemistry. NSCs were fixed in $4 \%$ paraformaldehyde (PF) (Sigma-Aldrich) for $1 \mathrm{~h}$ at $4^{\circ} \mathrm{C}$, followed by washing in phosphate-buffered saline 

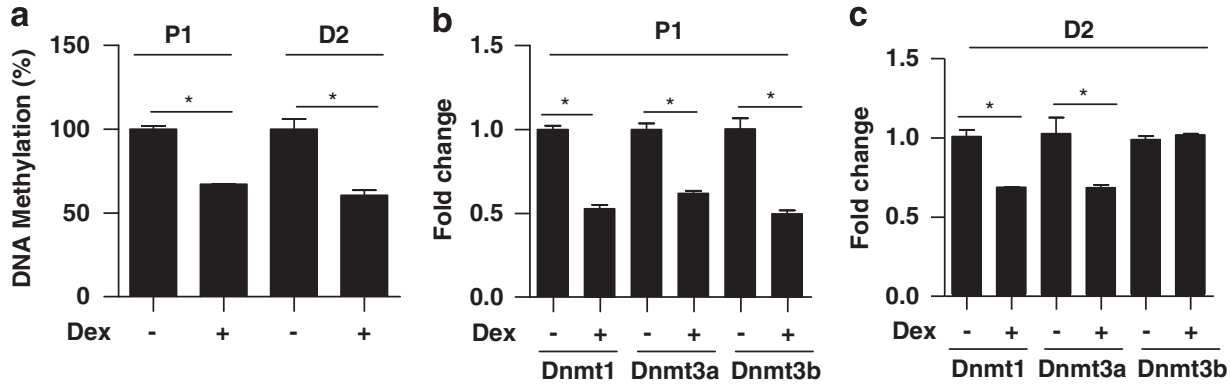

Figure 7 Dex modifies global DNA methylation. Global levels of cytosine methylation were decreased in P1 and D2 Dex-exposed NSCs (a) with concomitant downregulation of methyltransferases Dnmt1 and Dnmt3a mRNA (b and $\mathbf{c})$. The expression level of Dnmt3b mRNA was downregulated only in P1 cells (b). Values are shown as mean \pm S.E.M. ${ }^{*} P<0.05$
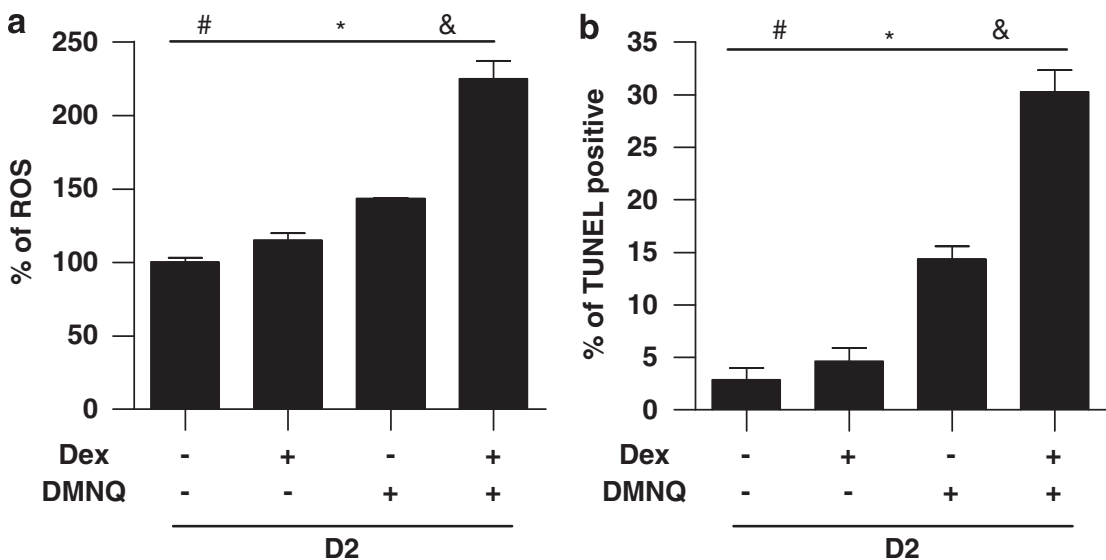

Figure 8 Dex increases NSC susceptibility to oxidative stress. D2 cells exposed to the oxidative stress inducer DMNQ for $8 \mathrm{~h}$ showed a significant increase in ROS accumulation (a) and an increased number of TUNEL-positive apoptotic cells, $24 \mathrm{~h}$ after DMNQ exposure (b). Values are shown as mean \pm S.E.M. ${ }^{*} P<0.05$

(PBS). Primary antibodies were diluted in PBS containing $0.3 \%$ Triton $\mathrm{X}-100$ and $0.5 \%$ bovine serum albumin (BSA, Boehringer Mannheim, Mannheim, Germany). NSCs were incubated with primary antibodies rabbit anti-Ki67 (1: 1000; Novocastra Laboratories Ltd, Wetzlar, Germany); mouse antinestin (1:200; Chemicon, Billerica, MA, USA); rabbit antiglial fibrillary acidic protein (GFAP, 1:800; Dakocytomation, Glostrup, Denmark); mouse antitubulin III (Tuj1, 1:400; Convance, Princeton, NJ, USA); and rabbit anti-HP1 $\gamma$ (1:1000; Cell Signaling, Danvers, MA, USA), overnight in a humid chamber at $4^{\circ} \mathrm{C}$. Cells were then rinsed with PBS and incubated with appropriate secondary FITC- or Texas-red-conjugated antibodies for $1 \mathrm{~h}$ at room temperature (RT) (1:200; Alexa, Invitrogen, Carlsbad, CA, USA). Cell nuclei were counterstained with Hoechst $33342(1 \mu \mathrm{g} / \mathrm{ml}$, SigmaAldrich). After rinsing with PBS, coverslips were mounted onto slides with Vectashield mounting medium (Vector Laboratories, Inc, Burlingame, CA, USA). Images were captured using an Olympus BX60 fluorescence microscope (Olympus, Tokyo, Japan) equipped with a Hamamatsu digital camera (C4742-95-10sc, Hamamatsu Photonics Norden AB, Solna, Sweden). All experiments were performed in triplicate and repeated at least three times. Semiquantitative analyses were performed by counting at least 100 cells/coverslip in triplicate.

Extraction of total RNA, cDNA synthesis and real-time PCR. Total RNA was isolated from NSCs using the RNeasy Mini Kit (Qiagen, VWR, Stockholm, Sweden). Integrity and concentration of extracted RNA were measured using a NanoDrop 1000 spectrophotometer (Thermo Scientific, Wilmington, DE, USA). cDNA was prepared using $1 \mu \mathrm{g}$ total RNA and $0.5 \mu \mathrm{g}$ of Oligo-dT primer following the instructions of the Superscript II first strand cDNA synthesis kit (Invitrogen Inc). Amplification reactions were performed with $1 \mu \mathrm{l}$ cDNA, SYBR Green Mix (Applied Biosystems, Stockholm, Sweden) and $0.2 \mu \mathrm{M}$ of forward and reverse primers. The reaction volume was adjusted to $25 \mu \mathrm{l}$ with DEPC water. Negative control reactions contained water instead of CDNA as template. q-PCR was performed using an $\mathrm{AB}$ Prism 7000 Sequence Detection System with SDS version 2.1 software (Applied Biosystems). The PCR cycle conditions were $50^{\circ} \mathrm{C}$ for $2 \mathrm{~min}, 95^{\circ} \mathrm{C}$ for $10 \mathrm{~min}, 95^{\circ} \mathrm{C}$ for $15 \mathrm{~s}$ and $60^{\circ} \mathrm{C}$ for $1 \mathrm{~min}$ (40 cycles). To evaluate the amplification of a specific sample, final melting curve (from $60^{\circ} \mathrm{C}$ up to $95^{\circ} \mathrm{C}$ ) was added under continuous fluorescence measurements. All expression values were normalized against the housekeeping gene hypoxanthine phosphoribosyltransferase (Hprt) $\left(\Delta C T=C T_{\text {target }}\right.$ gene $\left.-C T_{\text {Hprt }}\right)$. Relative expression levels were calculated as $\Delta \Delta C T=\Delta \mathrm{CT}_{\text {Dex }}-\Delta \mathrm{CT}_{\text {control }}$ and relative expression changes were calculated as $2^{-\Delta \Delta C T}$. Representative values are shown as mean \pm S.E.M. Statistical significance was set to $P<0.05$. All experiments were conducted in triplicate and repeated at least three times. PCR primer sequences are available in Supplementary Table S1.

siRNA nucleofection. siRNA targeting rat GR (5'-CAUGUUAGGUGGG CGUCAA- $\left.3^{\prime}\right)$ and negative siRNA control sequence (5'-UUGACGCCCACCUA ACAUG- $3^{\prime}$ ) were purchased from Qiagen. siRNA was delivered by using a Nucleofactor device and the Nucleofactor kit according to the supplier's protocol (Amaxa, Lonza, Switzerland). At $3 \mathrm{~h}$ after nucleofection, NSCs were treated with $1 \mu \mathrm{M}$ Dex for $24 \mathrm{~h}$. Thereafter, the cells were harvested for gene expression analysis. Knockdown efficiency was analysed by q-PCR analysis. All experiments were carried out in triplicate and repeated at least three times.

Global DNA methylation assay. DNA was prepared using the GeneElute Mammalian Genomic DNA Miniprep Kit (Sigma-Aldrich) according to the manufacturer's instructions. DNA quality and concentration were measured by a NanoDrop 1000 spectrophotometer (Thermo Scientific). Global DNA methylation was determined with a MethylAMP Global DNA Methylation Quantification Kit 
(Epigenetek, New York, NY, USA) as instructed by the manufacturer, including methylated DNA standard as positive control. Global cytosine methylation levels were determined by measuring optical density (OD) in a microplate reader at $450 \mathrm{~nm}$. The percentage of cytosine methylation was calculated in Dex-treated cells relative to untreated control cells, according to the manufacturer's instructions.

Measurement of intracellular ROS levels. D2 NSCs were seeded in 96well plates. At $72 \mathrm{~h}$ after seeding, cells were exposed to $3 \mu \mathrm{M}$ DMNQ for $8 \mathrm{~h}$, then washed with HBSS buffer and incubated with $10 \mu \mathrm{M}$ 5-(and-6)-carboxy-2', $7^{\prime}$ dichlorodihydrofluorescein diacetate (carboxy- $\mathrm{H}_{2} \mathrm{DCFDA}$ ) (Image-iT Live Green ROS Detection Kit, Molecular Probes, Carlsbad, CA, USA) for 30 min under $5 \% \mathrm{CO}^{2}$ atmosphere. The non-fluorescent carboxy- $\mathrm{H}_{2}$ DCFDA permeates live cells and is deacetylated by nonspecific intracellular esterases. In the presence of ROS, the reduced fluorescein compound is oxidized and emits bright green. The fluorescence intensity of cells from each well was analysed by Fluoroscan Ascent FL (Thermo Scientific) at an excitation wavelength of $495 \mathrm{~nm}$ and emission wavelength of $529 \mathrm{~nm}$, followed by determination of the protein content of the respective well using the NanoDrop 1000 spectrophotometer (Thermo Scientific). The data are expressed as fluorescence intensity related to the protein content. All experiments were performed in triplicate and repeated at least three times.

Detection of apoptotic cells. To evaluate the nuclear morphology, NSCs (P1, D2 and D3) were grown on poly-L-ornithine/fibronectin-coated coverslips and fixed in $4 \% \mathrm{PF}$ for $1 \mathrm{~h}$ at $4^{\circ} \mathrm{C}$. After washing with PBS, cells were stained with Hoechst $33342(1 \mu \mathrm{g} / \mathrm{ml})$ for $5 \mathrm{~min}$ at RT, and then rinsed with PBS. Apoptotic cells were identified by the condensed chromatin. TUNEL assay was performed to detect single-strand DNA breaks. NSCs were fixed as described above and then incubated with TUNEL reaction mixture $\left(0.07 \%\right.$ Triton $\mathrm{X}-100,2.5 \mathrm{mM} \mathrm{CoCl}_{2}, 5 \mu \mathrm{M}$ fluorescein12-UTP, $5 \mathrm{U} / \mu \mathrm{l}$ terminal transferase, $0.2 \mathrm{mM}$ potassiumcacodylate, $0.25 \mathrm{mg} / \mathrm{ml}$ BSA, $25 \mathrm{mM}$ Tris- $\mathrm{HCl} \mathrm{pH} \mathrm{6.6)} \mathrm{(Roche,} \mathrm{Bromma,} \mathrm{Sweden)} \mathrm{at} 37^{\circ} \mathrm{C}$ for $1 \mathrm{~h}$. After mounting, cells were analysed with a fluorescent microscope and at least 100 nuclei were counted per coverslip. All experiments were performed in triplicate and repeated at least three times.

Statistical analysis. All data are presented as mean \pm standard error of the mean. Quantitative data were analysed by one-way analysis of variance (ANOVA), followed by Bonferroni's post hoc test. The threshold of statistical significance was set at 0.05 for all analyses.

\section{Conflict of interest}

The authors declare no conflict of interest.

Acknowledgements. This study was supported by the Swedish Research Council and Karolinska Institutet.

1. Fowden AL, Forhead AJ. Endocrine mechanisms of intrauterine programming. Reproduction 2004; 127: 515-526.

2. Barker DJ. Outcome of low birth weight. Horm Res 1994; 42: 223-230.

3. Strang-Karlsson S, Räikkonen K, Pesonen AK, Kajantie E, Paavonen EJ, Lahti J et al. Very low birth weight and behavioral symptoms of attention deficit hyperactivity disorder in young adulthood: the Helsinki study of very-low-birth-weight adults. Am J Psychiatry 2008; 165: $1345-1353$

4. Räikkönen K, Pesonen AK, Heinonen K, Kajantie E, Hovi $P$, Jarvenpaa AL et al. Depression in young adults with very low birth weight: the Helsinki study of very low-birthweight adults. Arch Gen Psychiatry 2008; 65: 290-296.

5. Seckl JR. Prenatal glucocorticoids and long-term programming. Eur J Endocrinol 2004; 151 (Suppl 3): U49-U62.

6. Buckingham JC. Glucocorticoids: exemplars of multi-tasking. Br J Pharmacol 2006; 147 (Suppl 1): S258-S268.

7. de Kloet ER, Joels M, Holsboer F. Stress and the brain: from adaptation to disease. Nat Rev Neurosci 2005; 6: 463-475.

8. McEwen BS. Physiology and neurobiology of stress and adaptation: central role of the brain. Physiol Rev 2007; 87: 873-904.

9. Lemaire V, Koehl M, Le Moal M, Abrous DN. Prenatal stress produces learning deficits associated with an inhibition of neurogenesis in the hippocampus. Proc Natl Acad Sci USA 2000; 97: 11032-11037.

10. Lucassen PJ, Bosch OJ, Jousma E, Kromer SA, Andrew R, Seckl JR et al. Prenatal stress reduces postnatal neurogenesis in rats selectively bred for high, but not low, anxiety: possible key role of placental 11beta-hydroxysteroid dehydrogenase type 2. Eur J Neurosci 2009; 29: 97-103.

11. Weinstock $M$. The long-term behavioural consequences of prenatal stress Neurosci Biobehav Rev 2008; 32: 1073-1086.

12. Zhao $\mathrm{C}$, Deng W, Gage FH. Mechanisms and functional implications of adult neurogenesis Cell 2008; 132: 645-660.

13. Wong EY, Herbert J. Raised circulating corticosterone inhibits neuronal differentiation of progenitor cells in the adult hippocampus. Neuroscience 2006; 137: 83-92.

14. Gould E, Tanapat P, McEwen BS, Flugge G, Fuchs E. Proliferation of granule cell precursors in the dentate gyrus of adult monkeys is diminished by stress. Proc Natl Acad Sci USA 1998; 95: 3168-3171.

15. Fenoglio KA, Brunson KL, Baram TZ. Hippocampal neuroplasticity induced by early-life stress: functional and molecular aspects. Front Neuroendocrinol 2006; 27: 180-192.

16. Ahlbom E, Gogvadze V, Chen M, Celsi G, Ceccatelli S. Prenatal exposure to high levels of glucocorticoids increases the susceptibility of cerebellar granule cells to oxidative stress-induced cell death. Proc Natl Acad Sci USA 2000; 97: 14726-14730.

17. Ceccatelli S, Tamm C, Zhang $Q$, Chen M. Mechanisms and modulation of neural cell damage induced by oxidative stress. Physiol Behav 2007; 92: 87-92.

18. Rakic P. A small step for the cell, a giant leap for mankind: a hypothesis of neocortical expansion during evolution. Trends Neurosci 1995; 18: 383-388.

19. Caviness Jr VS, Takahashi T, Nowakowski RS. Numbers, time and neocortical neuronogenesis: a general developmental and evolutionary model. Trends Neurosci 1995; 18: 379-383.

20. Haydar TF. Advanced microscopic imaging methods to investigate cortical development and the etiology of mental retardation. Ment Retard Dev Disabil Res Rev 2005; 11: 303-316.

21. Flagstad P, Glenthoj BY, Didriksen M. Cognitive deficits caused by late gestational disruption of neurogenesis in rats: a preclinical model of schizophrenia. Neuropsychopharmacology 2005; 30: 250-260.

22. Sundberg M, Savola S, Hienola A, Korhonen L, Lindholm D. Glucocorticoid hormones decrease proliferation of embryonic neural stem cells through ubiquitin-mediated degradation of cyclin D1. J Neurosci 2006; 26: 5402-5410.

23. Stein GH, Drullinger LF, Soulard A, Dulic V. Differential roles for cyclin-dependent kinase inhibitors p21 and p16 in the mechanisms of senescence and differentiation in human fibroblasts. Mol Cell Biol 1999; 19: 2109-2117.

24. Herbig U, Jobling WA, Chen BP, Chen DJ, Sedivy JM. Telomere shortening triggers senescence of human cells through a pathway involving ATM, p53, and p21(CIP1), but not p16(INK4a). Mol Cell 2004; 14: 501-513.

25. Datson NA, Morsink MC, Meijer OC, de Kloet ER. Central corticosteroid actions: search for gene targets. Eur J Pharmacol 2008; 583: 272-289.

26. Cram EJ, Ramos RA, Wang EC, Cha HH, Nishio Y, Firestone GL. Role of the CCAAT/ enhancer binding protein-alpha transcription factor in the glucocorticoid stimulation of p21waf1/cip1 gene promoter activity in growth-arrested rat hepatoma cells. J Biol Chem 1998; 273: 2008-2014.

27. Cha HH, Cram EJ, Wang EC, Huang AJ, Kasler HG, Firestone GL. Glucocorticoids stimulate p21 gene expression by targeting multiple transcriptional elements within steroid responsive region of the p21waf1/cip1 promoter in rat hepatoma cells. J Biol Chem 1998; 273: 1998-2007.

28. Herbig U, Sedivy JM. Regulation of growth arrest in senescence: telomere damage is not the end of the story. Mech Ageing Dev 2006; 127: 16-24.

29. Chang BD, Watanabe K, Broude EV, Fang J, Poole JC, Kalinichenko TV et al. Effects of p21Waf1/Cip1/Sdi1 on cellular gene expression: implications for carcinogenesis, senescence, and age-related diseases. Proc Natl Acad Sci USA 2000; 97: 4291-4296.

30. Narita M. Cellular senescence and chromatin organisation. Br J Cancer 2007; 96 686-691.

31. Park IK, Morrison SJ, Clarke MF. Bmi1, stem cells, and senescence regulation $J$ Clin Invest 2004; 113: 175-179.

32. Passos JF, Saretzki G, Ahmed S, Nelson G, Richter T, Peters $\mathrm{H}$ et al. Mitochondrial dysfunction accounts for the stochastic heterogeneity in telomere-dependent senescence. PLOS Biol 2007; 5: e110.

33. Ksiazek K, Passos JF, Olijslagers S, von Zglinicki T. Mitochondrial dysfunction is a possible cause of accelerated senescence of mesothelial cells exposed to high glucose. Biochem Biophys Res Commun 2008; 366: 793-799.

34. Xu J, Shi C, Li Q, Wu J, Forster EL, Yew DT. Mitochondrial dysfunction in platelets and hippocampi of senescence-accelerated mice. J Bioenerg Biomembr 2007; 39: 195-202.

35. Moiseeva O, Bourdeau V, Roux A, Deschenes-Simard X, Ferbeyre G. Mitochondrial dysfunction contributes to oncogene-induced senescence. Mol Cell Biol 2009; 29: 4495-4507.

36. Navarro A, Boveris A. The mitochondrial energy transduction system and the aging process. Am J Physiol Cell Physiol 2007; 292: C670-C686.

37. Demonacos CV, Karayanni N, Hatzoglou E, Tsiriyiotis C, Spandidos DA, Sekeris CE. Mitochondrial genes as sites of primary action of steroid hormones. Steroids 1996; 61: 226-232.

38. Datson NA, van der Perk J, de Kloet ER, Vreugdenhil E. Identification of corticosteroidresponsive genes in rat hippocampus using serial analysis of gene expression. Eur J Neurosci 2001; 14: 675-689. 
39. Ilkhanizadeh S, Teixeira Al, Hermanson O. Inkjet printing of macromolecules on hydrogels to steer neural stem cell differentiation. Biomaterials 2007; 28: 3936-3943.

40. Tamm C, Duckworth J, Hermanson O, Ceccatelli S. High susceptibility of neural stem cells to methylmercury toxicity: effects on cell survival and neuronal differentiation. $J$ Neurochem 2006; 97: 69-78.
Cell Death and Disease is an open-access journal SOMERILHIS RESERVED published by Nature Publishing Group. This work is
licensed under the Creative Commons Attribution-Noncommercial-No Derivative Works 3.0 Unported License. To view a copy of this license, visit http://creativecommons.org/licenses/by-nc-nd/3.0/

Supplementary Information accompanies the paper on Cell Death and Disease website (http://www.nature.com/cddis) 